\title{
Modeling the Chemistry of Gaseous Cations Derived from Tricarbon Dioxide
}

Steven M. Schildcrout

Department of Chemical and Biological Sciences

Youngstown State University, Youngstown, OH 44555 USA

\section{Supporting Information}

\section{List of Tables}

Table S1. Absolute thermochemical parameters of cations and neutral species at optimized geometries

Table S2. Mulliken spin densities of doublet $\mathrm{C}_{p} \mathrm{O}_{3}{ }^{+}$

Table S3. Computed $\Delta E_{Q D}=E_{Q}-E_{D}$ between optimized quartets and doublets for oligocarbon trioxide ions $\mathrm{C}_{p} \mathrm{O}_{3}{ }^{+}$

Table S4. Optimized bond lengths $(R / p m)$ and dipole moments of quartet $\mathrm{C}_{p} \mathrm{O}_{3}{ }^{+}$ions.

Table S5. Mulliken spin densities of quartet $\mathrm{C}_{p} \mathrm{O}_{3}{ }^{+}$

Table S6. Mulliken spin densities of doublet $\mathrm{C}_{p} \mathrm{O}^{+}$

Table S7. Mulliken spin densities of doublet $\mathrm{C}_{p} \mathrm{O}_{2}{ }^{+}$

Table S8. Computed $\Delta E_{\mathrm{QD}}$ between optimized quartets and doublets for oligocarbon monoxide and dioxide ions (as Fig 1)

Table S9. Mulliken spin densities of quartet $\mathrm{C}_{p} \mathrm{O}^{+}$

Table S10. Optimized bond lengths and dipole moments of quartet $\mathrm{C}_{p} \mathrm{O}^{+}$

Table S11. Mulliken spin densities of quartet $\mathrm{C}_{p} \mathrm{O}_{2}^{+}$

Table S12. Optimized bond lengths and dipole moments of quartet $\mathrm{C}_{p} \mathrm{O}_{2}{ }^{+}$

Table S13. Monocarbon-insertion energetics for doublet $\mathrm{C}_{p} \mathrm{O}_{3}{ }^{+}$.

Table S14. Computed $\Delta E_{\mathrm{TS}}$ between optimized triplets and singlets of neutral oligocarbon monoxides and dioxides (as Fig 3)

Table S15. Computed vertical electron-attachment and CT (Equation 10) energies for $\mathrm{C}_{p} \mathrm{O}^{+}$giving neutral $\mathrm{C}_{p} \mathrm{O}$ in most-stable spin state (Compare Table 14)

Table S16. Optimized bond lengths and dipole moments of neutral $\mathrm{C}_{p} \mathrm{O}$ molecules

Table S17. Mulliken spin densities of triplet $\mathrm{C}_{p} \mathrm{O}$ molecules

Table S18. Computed vertical electron-attachment and CT (Equation 10) energies for $\mathrm{C}_{p} \mathrm{O}_{2}{ }^{+}$ giving neutral $\mathrm{C}_{p} \mathrm{O}_{2}$ in most-stable spin state (Compare Table 15)

Table S19. Mulliken spin densities of triplet $\mathrm{C}_{p} \mathrm{O}_{2}$ molecules

Table S20. Computed vertical electron-attachment and CT (Equation 10) energies for $\mathrm{C}_{p} \mathrm{O}_{3}{ }^{+}$. giving neutral $\mathrm{C}_{p} \mathrm{O}_{3}$ in the indicated spin state (Compare Table 17)

Table S21. Mulliken spin densities of triplet $\mathrm{C}_{p} \mathrm{O}_{3}$ molecules 


\section{Supporting information}

Table S1. Absolute thermochemical parameters of cations and neutral species at optimized geometries by B3LYP/jun-cc-pVTZ.

For neutral species, $E$ is shown also at the optimized geometry of the cation for computation of vertical electron-attachment energy. Except as noted, geometries are linear, $\mathrm{C}_{\infty v}$ for monoxides and $D_{\infty h}$ for dioxides. The most stable isomers or spin states are in bold. 1 au (atomic unit) = 1 hartree $=2625.5 \mathrm{~kJ} / \mathrm{mol}=27.211 \mathrm{eV}$

\begin{tabular}{|c|c|c|c|c|c|c|c|}
\hline Formula & $2 S+1$ & E/au & $\mathrm{H}_{298^{\circ}} / \mathrm{au}$ & $\mathrm{S}_{298^{\circ} /(\mathrm{J} / \mathrm{mol} \mathrm{K})}$ & $G_{298^{\circ}} / \mathrm{au}$ & Geometry & E/au @ ion geom \\
\hline $\mathrm{CO}^{+}$ & 2 & -112.837856 & -112.829331 & 203.0 & -112.852382 & & \\
\hline $\mathrm{CCO}^{+}$ & 2 & -150.901701 & -150.889487 & 235.8 & -150.916260 & & \\
\hline $\mathrm{CCO}^{+}$ & 4 & -150.894307 & -150.880615 & 232.1 & -150.906973 & & \\
\hline $\mathrm{COC}^{+}$ & 2 & -150.846617 & -150.836336 & 257.0 & -150.865525 & $C_{\infty v}$ & \\
\hline $\mathrm{COC}^{+}$ & 4 & -150.747411 & -150.735735 & 228.2 & -150.761648 & $\mathrm{D}_{\infty \mathrm{h}}$ & \\
\hline $\mathrm{C}_{2} \mathrm{O}^{+}$ & 2 & -150.837713 & -150.828219 & 245.8 & -150.856133 & cyclo $C_{2 v}$ & \\
\hline $\mathrm{C}_{2} \mathrm{O}^{+}$ & 4 & unstable & & & & cyclo $C_{s}$ & \\
\hline $\mathrm{C}_{3} \mathrm{O}^{+}$ & 2 & -189.062482 & -189.041583 & 254.8 & -189.070518 & & \\
\hline $\mathrm{C}_{3} \mathrm{O}^{+}$ & 4 & -188.925805 & -188.909417 & 280.8 & -188.941306 & $C_{s}$ & \\
\hline $\mathrm{C}_{4} \mathrm{O}^{+}$ & 2 & -227.143271 & -227.118155 & 284.8 & -227.150498 & & \\
\hline $\mathrm{C}_{4} \mathrm{O}^{+}$ & 4 & -227.133456 & -227.107989 & 284.5 & -227.140297 & & \\
\hline $\mathrm{C}_{5} \mathrm{O}^{+}$ & 2 & -265.272683 & -265.240260 & 286.1 & -265.272743 & $\mathrm{C}_{\mathrm{s}}$ & \\
\hline $\mathrm{C}_{5} \mathrm{O}^{+}$ & 4 & -265.175903 & -265.147737 & 332.3 & -265.185477 & $\mathrm{C}_{\mathrm{s}}$ & \\
\hline $\mathrm{C}_{6} \mathrm{O}^{+}$ & 2 & -303.366040 & -303.328634 & 330.8 & -303.366244 & & \\
\hline $\mathrm{C}_{6} \mathrm{O}^{+}$ & 4 & -303.348805 & -303.311524 & 331.2 & -303.349136 & & \\
\hline $\mathrm{C}_{7} \mathrm{O}^{+}$ & 2 & -341.475439 & -341.433649 & 361.0 & -341.474637 & $C_{s}$ & \\
\hline $\mathrm{C}_{7} \mathrm{O}^{+}$ & 4 & -341.410285 & -341.369748 & 372.5 & -341.412042 & $\mathrm{C}_{\mathrm{s}}$ & \\
\hline $\mathrm{CO}_{2}^{+}$ & 2 & -188.155722 & -188.143250 & 221.7 & -188.168422 & & \\
\hline $\mathrm{C}_{2} \mathrm{O}_{2}{ }^{+}$ & 2 & -226.298642 & -226.280057 & 268.5 & -226.310550 & $\mathrm{C}_{2 \mathrm{~h}}$ & \\
\hline $\mathrm{C}_{2} \mathrm{O}_{2}^{+}$ & 4 & -226.152011 & -226.134311 & 278.6 & -226.165951 & $\mathrm{C}_{s}$ & \\
\hline $\mathrm{C}_{3} \mathrm{O}_{2}^{+}$ & 2 & -264.433621 & -264.407924 & 286.5 & -264.440460 & $C_{2 v}$ & \\
\hline $\mathrm{C}_{3} \mathrm{O}_{2}^{+}$ & 4 & -264.318396 & -264.295225 & 317.8 & -264.331317 & $\mathrm{C}_{s}$ & \\
\hline $\mathrm{C}_{4} \mathrm{O}_{2}{ }^{+}$ & 2 & -302.540213 & -302.508271 & 266.4 & -302.538521 & & \\
\hline $\mathrm{C}_{4} \mathrm{O}_{2}{ }^{+}$ & 4 & -302.449207 & -302.418736 & 317.8 & -302.454828 & & \\
\hline $\mathrm{C}_{5} \mathrm{O}_{2}^{+}$ & 2 & -340.654879 & -340.616642 & 324.2 & -340.653460 & & \\
\hline $\mathrm{C}_{5} \mathrm{O}_{2}^{+}$ & 4 & -340.558978 & -340.523809 & 358.4 & -340.564512 & $\mathrm{C}_{\mathrm{s}}$ & \\
\hline $\mathrm{C}_{6} \mathrm{O}_{2}{ }^{+}$ & 2 & -378.756313 & -378.711561 & 345.6 & -378.750807 & & \\
\hline $\mathrm{C}_{6} \mathrm{O}_{2}{ }^{+}$ & 4 & -378.687693 & -378.645021 & 358.2 & -378.685697 & & \\
\hline $\mathrm{C}_{7} \mathrm{O}_{2}{ }^{+}$ & 2 & -416.862005 & -416.811637 & 368.5 & -416.853483 & & \\
\hline $\mathrm{C}_{7} \mathrm{O}_{2}^{+}$ & 4 & -416.789459 & -416.740852 & 382.0 & -416.784232 & & \\
\hline $\mathrm{C}_{4} \mathrm{O}_{3}{ }^{+}$ & 2 & -377.822112 & -377.785387 & 344.5 & -377.824508 & $C_{s}$ & \\
\hline $\mathrm{C}_{4} \mathrm{O}_{3}{ }^{+}$ & 4 & -377.701325 & -377.667788 & 377.7 & -377.710678 & $\mathrm{C}_{2 \mathrm{v}}$ & \\
\hline $\mathrm{C}_{5} \mathrm{O}_{3}{ }^{+}\left(\mathrm{d}^{+}\right)$ & 2 & -415.914008 & -415.870862 & 368.2 & -415.912671 & $C_{s}$ & \\
\hline $\mathrm{C}_{5} \mathrm{O}_{3}{ }^{+}\left(\mathrm{e}^{+}\right)$ & 2 & -415.896130 & -415.853256 & 356.2 & -415.893705 & $\mathrm{C}_{2 \mathrm{v}}$ & \\
\hline $\mathrm{C}_{5} \mathrm{O}_{3}{ }^{+}$ & 4 & -415.850588 & -415.809387 & 383.1 & -415.852894 & $C_{s}$ & \\
\hline $\mathrm{C}_{6} \mathrm{O}_{3}{ }^{+}$ & 2 & -454.020916 & -453.972145 & 394.3 & -454.016916 & $C_{s}$ & \\
\hline $\mathrm{C}_{6} \mathrm{O}_{3}{ }^{+}$ & 4 & -453.927749 & -453.881651 & 410.4 & -453.928250 & $C_{s}$ & \\
\hline
\end{tabular}




\begin{tabular}{|c|c|c|c|c|c|c|c|}
\hline $\mathrm{C}_{7} \mathrm{O}_{3}{ }^{+}\left(\mathrm{f}^{+}\right)$ & 2 & -492.113892 & -492.059107 & 433.4 & -492.108321 & $\sim \mathrm{C}_{2 v}$ & \\
\hline $\mathrm{C}_{7} \mathrm{O}_{3}{ }^{+}$ & 2 & -492.110959 & -492.055598 & 417.4 & -492.103003 & $C_{s}$ & \\
\hline $\mathrm{C}_{7} \mathrm{O}_{3}{ }^{+}$ & 4 & -492.073969 & -492.020645 & 427.2 & -492.069162 & $\mathrm{C}_{\mathrm{s}}$ & \\
\hline $\mathrm{C}_{6} \mathrm{O}_{4}{ }^{+}\left(\mathrm{c}^{+}\right)$ & 2 & -529.303217 & -529.248670 & 388.9 & -529.292836 & $C_{2 v}$ & \\
\hline $\mathrm{C}_{6} \mathrm{O}_{4}^{+}\left(\mathbf{b}^{+}\right)$ & 2 & -529.290339 & -529.236426 & 386.5 & -529.280321 & $\mathrm{D}_{2 \mathrm{~h}}$ & \\
\hline $\mathrm{C}_{6} \mathrm{O}_{4}{ }^{+}\left(\mathrm{a}^{+}\right)$ & 2 & -529.280448 & -529.226172 & 425.5 & -529.274496 & $\mathrm{C}_{1}$ & \\
\hline $\mathrm{CO}$ & 1 & -113.358681 & -113.350344 & 197.4 & -113.372768 & & -113.358064 \\
\hline $\mathrm{CCO}$ & 1 & -151.278662 & -151.265715 & 224.2 & -151.291177 & & -151.261717 \\
\hline $\mathrm{CCO}$ & 3 & -151.318592 & -151.305725 & 233.3 & -151.332216 & & -151.303054 \\
\hline $\mathrm{COC}$ & 1 & -151.173987 & -151.163166 & 219.4 & -151.188075 & & -151.160172 \\
\hline $\mathrm{COC}$ & 3 & -151.214865 & -151.204510 & 239.7 & -151.230303 & & -151.207303 \\
\hline $\mathrm{C}_{2} \mathrm{O}$ & 1 & -151.249418 & -151.238027 & 233.0 & -151.264498 & cyclo $C_{2 v}$ & -151.239312 \\
\hline $\mathrm{C}_{2} \mathrm{O}$ & 3 & unstable & & & & cyclo & -151.168432 \\
\hline $\mathrm{C}_{3} \mathrm{O}$ & 1 & -189.467409 & -189.447035 & 253.0 & -189.475766 & & -189.459820 \\
\hline $\mathrm{C}_{3} \mathrm{O}$ & 3 & -189.358028 & -189.340717 & 276.4 & -189.372110 & $\mathrm{C}_{s}$ & \\
\hline $\mathrm{C}_{4} \mathrm{O}$ & 1 & -227.484579 & -189.460256 & 290.2 & -227.493211 & $C_{s}$ & \\
\hline $\mathrm{C}_{4} \mathrm{O}$ & 3 & -227.510244 & -227.484993 & 283.8 & -227.517227 & & -227.502283 \\
\hline $\mathrm{C}_{5} \mathrm{O}$ & 1 & -265.641355 & -265.608929 & 301.2 & -265.643132 & & -265.636842 \\
\hline $\mathrm{C}_{5} \mathrm{O}$ & 3 & -265.556827 & -265.528002 & 329.0 & -265.565360 & $\mathrm{C}_{s}$ & \\
\hline $\mathrm{C}_{6} \mathrm{O}$ & 1 & -303.682929 & -303.644503 & 328.5 & -303.682809 & & \\
\hline $\mathrm{C}_{6} \mathrm{O}$ & 3 & -303.702160 & -303.664837 & 331.2 & -303.702448 & & -303.695319 \\
\hline $\mathrm{C}_{7} \mathrm{O}$ & 1 & -341.821932 & -341.777515 & 347.8 & -341.817012 & & -341.817608 \\
\hline $\mathrm{C}_{7} \mathrm{O}$ & 3 & -341.753066 & -341.712503 & 365.0 & -341.753950 & $C_{s}$ & \\
\hline $\mathrm{CO}_{2}$ & 1 & -188.663094 & -188.647870 & 213.6 & -188.672122 & & -188.662575 \\
\hline $\mathrm{C}_{2} \mathrm{O}_{2}$ & 3 & -226.655708 & -226.636389 & 257.4 & -226.665612 & & -226.629257 \\
\hline $\mathrm{C}_{2} \mathrm{O}_{2}$ & 1 & unstable & & & & & \\
\hline $\mathrm{C}_{3} \mathrm{O}_{2}$ & 1 & -264.827693 & -264.800365 & 279.2 & -264.832068 & & -264.822698 \\
\hline $\mathrm{C}_{3} \mathrm{O}_{2}$ & 3 & -264.708690 & -264.684719 & 310.8 & -264.720013 & $\mathrm{C}_{1}$ & \\
\hline $\mathrm{C}_{4} \mathrm{O}_{2}$ & 1 & -302.843727 & -302.812955 & 308.0 & -302.847937 & $\mathrm{C}_{2 \mathrm{~h}}$ & \\
\hline $\mathrm{C}_{4} \mathrm{O}_{2}$ & 3 & -302.864248 & -302.832345 & 301.4 & -302.866576 & & -302.854950 \\
\hline $\mathrm{C}_{5} \mathrm{O}_{2}$ & 1 & -341.001966 & -340.962700 & 318.9 & -340.998918 & & -340.998254 \\
\hline $\mathrm{C}_{5} \mathrm{O}_{2}$ & 3 & -340.903512 & -340.868494 & 345.8 & -340.907761 & $C_{2 v}$ & \\
\hline $\mathrm{C}_{6} \mathrm{O}_{2}$ & 1 & -379.041819 & -378.997727 & 351.7 & -379.037671 & & \\
\hline $\mathrm{C}_{6} \mathrm{O}_{2}$ & 3 & -379.058258 & -379.014242 & 349.0 & -379.053879 & & -379.050032 \\
\hline $\mathrm{C}_{7} \mathrm{O}_{2}$ & 1 & -417.181760 & -417.130469 & 364.8 & -417.171895 & & -417.178186 \\
\hline $\mathrm{C}_{7} \mathrm{O}_{2}$ & 3 & -417.113729 & -417.065256 & 378.2 & -417.108206 & & \\
\hline $\mathrm{C}_{4} \mathrm{O}_{3}$ & 3 & -378.100167 & -378.064761 & 346.9 & -378.104165 & $C_{2 v}$ & -378.064297 \\
\hline $\mathrm{C}_{4} \mathrm{O}_{3}$ & 1 & unstable & & & & & -378.094892 \\
\hline $\mathrm{C}_{5} \mathrm{O}_{3}(\mathrm{e})$ & 1 & -416.213684 & -416.170380 & 361.6 & -416.211896 & $C_{2 v}$ & \\
\hline $\mathrm{C}_{5} \mathrm{O}_{3}(\mathrm{~d})$ & 1 & -416.195274 & -416.153010 & 378.2 & -416.195956 & $C_{s}$ & -416.189040 \\
\hline $\mathrm{C}_{5} \mathrm{O}_{3}(\mathbf{d})$ & 3 & -416.192580 & -416.151151 & 377.6 & -416.194035 & $C_{s}$ & -416.162041 \\
\hline $\mathrm{C}_{6} \mathrm{O}_{3}$ & 3 & -454.280746 & -454.233194 & 399.6 & -454.278572 & $\mathrm{C}_{\mathrm{s}}$ & -454.255923 \\
\hline $\mathrm{C}_{6} \mathrm{O}_{3}$ & 1 & unstable & & & & & -454.276951 \\
\hline $\mathrm{C}_{7} \mathrm{O}_{3}(\mathrm{f})$ & 3 & -492.382444 & -492.328817 & 428.7 & -492.377503 & $C_{s}$ & -492.351871 \\
\hline $\mathrm{C}_{7} \mathrm{O}_{3}(\mathbf{f})$ & 1 & -492.378432 & -492.324221 & 427.6 & -492.372774 & $C_{s}$ & \\
\hline
\end{tabular}


Table S2. Mulliken spin densities ${ }^{\mathrm{a}}$ of doublet $\mathrm{C}_{p} \mathrm{O}_{3}{ }^{+}$

\begin{tabular}{|c|c|c|c|c|c|c|c|c|c|c|}
\hline$p^{\mathrm{b}}$ & $\mathrm{O}\left(\mathrm{C} 1^{\prime}\right)$ & $\mathrm{C}^{\prime}$ & $\mathrm{O}(\mathrm{C} 1)$ & $\mathrm{C} 1$ & $\mathrm{C} 2$ & $\mathrm{C} 3$ & $\mathrm{C} 4$ & $\mathrm{C} 5$ & $\mathrm{C} 6$ & $\mathrm{O}[\mathrm{C}(p-1)]$ \\
\hline 4 & 0.21 & $\mathbf{0 . 4 8}$ & 0.02 & 0.01 & 0.05 & 0.12 & -- & -- & -- & 0.11 \\
\hline 5 & 0.03 & 0.03 & 0.03 & 0.03 & -0.02 & $\mathbf{0 . 5 8}$ & 0.04 & -- & -- & 0.27 \\
\hline 6 & 0.19 & $\mathbf{0 . 4 2}$ & 0.01 & 0.01 & 0.08 & 0.03 & 0.12 & 0.06 & -- & 0.08 \\
\hline 7 & 0.02 & 0.02 & 0.02 & 0.02 & 0.00 & $\mathbf{0 . 3 8}$ & -0.14 & $\mathbf{0 . 4 3}$ & 0.04 & 0.21 \\
\hline
\end{tabular}

${ }^{a}$ Principal densities are bolded.

${ }^{\mathrm{b}}$ All are planar $\mathrm{C}_{\mathrm{s}}$.

Table S3. Computed $\Delta E_{Q D}=E_{Q}-E_{D}$ between optimized quartets and doublets for oligocarbon trioxide ions $\mathrm{C}_{p} \mathrm{O}_{3}{ }^{+}$

\begin{tabular}{|c|c|}
\hline$p$ & $\Delta E_{Q D} / \mathrm{eV}$ \\
\hline 4 & 3.287 \\
\hline 5 & 1.726 \\
\hline 6 & 2.535 \\
\hline 7 & 1.086 \\
\hline
\end{tabular}

Table S4. Optimized bond lengths $(R / p m)$ and dipole moments of quartet $\mathrm{C}_{p} \mathrm{O}_{3}{ }^{+}$ions.

\begin{tabular}{|c|c|c|c|c|c|c|c|c|c|c|}
\hline$p$ & C1-O & C1-C2 & C1 $^{\prime}-0$ & C1 $^{\prime}-C 2$ & C2-C3 & C3-C4 & C4-C5 & C5-C6 & C $(p-1)-O$ & $\mu / D$ \\
\hline $4^{\mathrm{a}}$ & 116.1 & 152.6 & 116.1 & 152.6 & 139.4 & & & & 112.9 & 0.82 \\
\hline $5^{\mathrm{b}}$ & 113.7 & 137.8 & 115.6 & 149.8 & 136.9 & 129.3 & & & 115.2 & 2.50 \\
\hline $6^{\mathrm{c}}$ & 113.8 & 143.3 & 114.8 & 148.7 & 136.0 & 130.8 & 130.7 & & 115.2 & 1.01 \\
\hline $7^{\mathrm{d}}$ & 113.9 & 137.3 & 115.9 & 148.7 & 137.2 & 125.5 & 128.0 & 129.7 & 114.7 & 3.22 \\
\hline
\end{tabular}

a ${ }^{4} \mathrm{~A}_{2}, A(\mathrm{C} 2, \mathrm{C} 1, \mathrm{O})=\mathrm{A}\left(\mathrm{C} 2, \mathrm{C} 1^{\prime}, \mathrm{O}\right)=129.0^{\circ}, \mathrm{A}(\mathrm{C} 1, \mathrm{C} 2, \mathrm{C} 3)=\mathrm{A}\left(\mathrm{C} 1^{\prime}, \mathrm{C} 2, \mathrm{C} 3\right)=121.5^{\circ}$

b ${ }^{4} \mathrm{~A}^{\prime \prime}, A(\mathrm{C} 2, \mathrm{C} 1, \mathrm{O})=163.7^{\circ}, A\left(\mathrm{C} 2, \mathrm{C} 1^{\prime}, \mathrm{O}\right)=136.1^{\circ}, A(\mathrm{C} 1, \mathrm{C} 2, \mathrm{C} 3)=125.2^{\circ}, A\left(\mathrm{C} 1^{\prime}, \mathrm{C} 2, \mathrm{C} 3\right)=119.0^{\circ}$

c ${ }^{4} \mathrm{~A}^{\prime \prime}, A(\mathrm{C} 2, \mathrm{C} 1, \mathrm{O})=152.9^{\circ}, A\left(\mathrm{C} 2, \mathrm{C} 1^{\prime}, \mathrm{O}\right)=141.8^{\circ}, A(\mathrm{C} 1, \mathrm{C} 2, \mathrm{C} 3)=124.1^{\circ}, A\left(\mathrm{C} 1^{\prime}, \mathrm{C} 2, \mathrm{C} 3\right)=124.0^{\circ}$, $\mathrm{A}(\mathrm{C} 2, \mathrm{C} 3, \mathrm{C} 4)=138.5^{\circ}, \mathrm{A}(\mathrm{C} 3, \mathrm{C} 4, \mathrm{C} 5)=158.9^{\circ}$

${ }^{4} \mathrm{~A}^{\prime \prime}, A(\mathrm{C} 2, \mathrm{C} 1, \mathrm{O})=164.2^{\circ}, A\left(\mathrm{C} 2, \mathrm{C} 1^{\prime}, \mathrm{O}\right)=137.0^{\circ}, A(\mathrm{C} 1, \mathrm{C} 2, \mathrm{C} 3)=124.5^{\circ}, A\left(\mathrm{C} 1^{\prime}, \mathrm{C} 2, \mathrm{C} 3\right)=120.4^{\circ}$

Table S5. Mulliken spin densities of quartet $\mathrm{C}_{p} \mathrm{O}_{3}{ }^{+}$

\begin{tabular}{|c|c|c|c|c|c|c|c|c|c|c|}
\hline$p^{\mathrm{a}}$ & $\mathrm{O}\left(\mathrm{C1}^{\prime}\right)$ & $\mathrm{C}^{\prime}$ & $\mathrm{O}(\mathrm{C} 1)$ & $\mathrm{C} 1$ & $\mathrm{C} 2$ & $\mathrm{C} 3$ & $\mathrm{C} 4$ & $\mathrm{C} 5$ & $\mathrm{C} 6$ & $\mathrm{O}[\mathrm{C}(p-1)]$ \\
\hline $4\left(\mathrm{C}_{2 \mathrm{v}}\right)$ & 0.39 & 0.44 & 0.39 & 0.44 & $\mathbf{0 . 9 3}$ & 0.14 & -- & -- & -- & 0.28 \\
\hline 5 & 0.29 & $\mathbf{0 . 5 4}$ & 0.21 & 0.23 & 0.08 & $\mathbf{1 . 0 3}$ & 0.11 & -- & -- & $\mathbf{0 . 5 1}$ \\
\hline 6 & 0.25 & 0.44 & 0.22 & 0.24 & 0.37 & 0.41 & $\mathbf{0 . 5 8}$ & 0.14 & -- & 0.36 \\
\hline 7 & 0.26 & $\mathbf{0 . 5 3}$ & 0.17 & 0.22 & 0.08 & $\mathbf{0 . 7 0}$ & -0.09 & $\mathbf{0 . 6 5}$ & 0.12 & 0.36 \\
\hline
\end{tabular}

a Planar $C_{s}$ except as noted 
Table S6. Mulliken spin densities of doublet $\mathrm{C}_{p} \mathrm{O}^{+}$

\begin{tabular}{|c|c|c|c|c|c|c|c|c|}
\hline$p^{\mathrm{a}}$ & $\mathrm{O}$ & $\mathrm{C} 1$ & $\mathrm{C} 2$ & $\mathrm{C} 3$ & $\mathrm{C} 4$ & $\mathrm{C} 5$ & $\mathrm{C} 6$ & $\mathrm{C7}$ \\
\hline 2 & 0.15 & 0.05 & $\mathbf{0 . 8 0}$ & -- & -- & -- & -- & -- \\
\hline 3 & -0.08 & 0.00 & 0.00 & $\mathbf{1 . 0 8}$ & -- & -- & -- & -- \\
\hline 4 & 0.15 & 0.09 & 0.19 & 0.03 & $\mathbf{0 . 5 4}$ & -- & -- & -- \\
\hline $5\left(\mathrm{C}_{s}\right)$ & -0.06 & -0.02 & -0.11 & 0.07 & 0.02 & $\mathbf{1 . 0 9}$ & -- & -- \\
\hline 6 & 0.12 & 0.07 & 0.16 & 0.04 & 0.15 & 0.04 & $\mathbf{0 . 4 2}$ & -- \\
\hline $7\left(C_{s}\right)$ & 0.10 & 0.00 & $\mathbf{0 . 2 3}$ & -0.13 & $\mathbf{0 . 3 1}$ & -0.15 & $\mathbf{0 . 2 8}$ & $\mathbf{0 . 3 7}$ \\
\hline
\end{tabular}

${ }^{a}$ Linear $C_{\infty v}$ except as noted

Table S7. Mulliken spin densities of doublet $\mathrm{C}_{p} \mathrm{O}_{2}{ }^{+}$

\begin{tabular}{|c|c|c|c|c|c|}
\hline$p^{\mathrm{a}}$ & $\mathrm{O}$ & $\mathrm{C} 1$ & $\mathrm{C} 2$ & $\mathrm{C} 3$ & $\mathrm{C} 4$ \\
\hline $2\left(\mathrm{C}_{2 \mathrm{~h}}\right)$ & 0.21 & 0.29 & -- & -- & -- \\
\hline $3\left(\mathrm{C}_{2 \mathrm{v}}\right)$ & 0.24 & -0.08 & $\mathbf{0 . 6 9}$ & -- & -- \\
\hline 4 & 0.18 & 0.16 & 0.16 & -- & -- \\
\hline 5 & 0.17 & -0.01 & $\mathbf{0 . 4 5}$ & -0.23 & -- \\
\hline 6 & 0.14 & 0.11 & 0.14 & 0.11 & -- \\
\hline 7 & 0.14 & 0.00 & $\mathbf{0 . 3 3}$ & -0.18 & $\mathbf{0 . 4 2}$ \\
\hline
\end{tabular}

${ }^{a}$ Linear $\mathrm{D}_{\infty \mathrm{h}}$ except as noted. Results that are equal by symmetry are shown only once.

Table S8. Computed $\Delta E_{\mathrm{QD}}$ between optimized quartets and doublets for oligocarbon monoxide and dioxide ions (as Fig 1)

\begin{tabular}{|c|c|c|}
\hline $\mathrm{C}_{p} \mathrm{O}_{q}{ }^{+}$ & \multicolumn{2}{|c|}{$\left(E_{Q^{-}} E_{\mathrm{D}}\right) / \mathrm{eV}$} \\
\hline$p$ & $q=1$ & $q=2$ \\
\hline 2 & 0.201 & 3.990 \\
\hline 3 & 3.719 & 3.135 \\
\hline 4 & 0.267 & 2.476 \\
\hline 5 & 2.634 & 2.610 \\
\hline 6 & 0.469 & 1.867 \\
\hline 7 & 1.773 & 1.974 \\
\hline
\end{tabular}

Table S9. Mulliken spin densities of quartet $\mathrm{C}_{p} \mathrm{O}^{+}$

\begin{tabular}{|c|c|c|c|c|c|c|c|c|}
\hline$p^{\mathrm{a}}$ & $\mathrm{O}$ & $\mathrm{C} 1$ & $\mathrm{C} 2$ & $\mathrm{C} 3$ & $\mathrm{C} 4$ & $\mathrm{C} 5$ & $\mathrm{C} 6$ & $\mathrm{C} 7$ \\
\hline 2 & 0.50 & 0.13 & $\mathbf{2 . 3 6}$ & -- & -- & -- & -- & -- \\
\hline $3\left(\mathrm{C}_{s}\right)$ & 0.36 & 0.21 & $\mathbf{0 . 7 3}$ & $\mathbf{1 . 7 0}$ & -- & -- & -- & -- \\
\hline 4 & 0.36 & 0.08 & $\mathbf{0 . 7 7}$ & -0.22 & $\mathbf{2 . 0 1}$ & -- & -- & -- \\
\hline $5\left(\mathrm{C}_{s}\right)$ & 0.42 & 0.13 & $\mathbf{0 . 8 1}$ & -0.02 & $\mathbf{0 . 7 1}$ & $\mathbf{0 . 9 4}$ & -- & -- \\
\hline 6 & 0.28 & 0.07 & $\mathbf{0 . 5 4}$ & -0.18 & $\mathbf{0 . 7 6}$ & -0.22 & $\mathbf{1 . 7 6}$ & -- \\
\hline $7\left(C_{s}\right)$ & 0.34 & 0.12 & $\mathbf{0 . 5 9}$ & -0.03 & $\mathbf{0 . 6 8}$ & 0.02 & 0.40 & $\mathbf{0 . 8 8}$ \\
\hline
\end{tabular}

${ }^{a}$ Linear $\mathrm{C}_{\infty \mathrm{v}}$ except as noted 
Table S10. Optimized bond lengths $(R / p m)$ and dipole moments of quartet $\mathrm{C}_{p} \mathrm{O}^{+}$

\begin{tabular}{|c|c|c|c|c|c|c|c|c|}
\hline$p^{\mathrm{a}}$ & $R(\mathrm{C} 1-\mathrm{O})$ & $R(\mathrm{C} 1-\mathrm{C} 2)$ & $R(\mathrm{C} 2-\mathrm{C} 3)$ & $R(\mathrm{C} 3-\mathrm{C} 4)$ & $R(\mathrm{C} 4-\mathrm{C} 5)$ & $R(\mathrm{C} 5-\mathrm{C} 6)$ & $R(\mathrm{C} 6-\mathrm{C} 7)$ & $\mu / \mathrm{D}$ \\
\hline 2 & 113.5 & 132.9 & -- & -- & -- & -- & -- & 3.14 \\
\hline $3\left(\mathrm{C}_{\mathrm{s}}\right)$ & 114.9 & 139.6 & 133.1 & -- & -- & -- & -- & 3.51 \\
\hline 4 & 113.7 & 131.0 & 127.1 & 126.5 & -- & -- & -- & 3.15 \\
\hline $5\left(\mathrm{C}_{s}\right)$ & 114.7 & 131.0 & 128.9 & 128.3 & 137.5 & -- & -- & 0.30 \\
\hline 6 & 114.0 & 130.5 & 125.7 & 128.0 & 129.3 & 124.5 & -- & 2.95 \\
\hline $7\left(\mathrm{C}_{\mathrm{s}}\right)$ & 114.6 & 130.6 & 126.7 & 128.0 & 129.1 & 127.1 & 133.7 & 0.69 \\
\hline
\end{tabular}

${ }^{a}$ Linear $\mathrm{C}_{\infty \mathrm{v}}$ except as noted. Angles are linear except as noted below:

$\mathrm{C}_{3} \mathrm{O}^{+} \mathrm{A}(\mathrm{O}, \mathrm{C} 1, \mathrm{C} 2)=147.9^{\circ}, \mathrm{A}(\mathrm{C} 1, \mathrm{C} 2, \mathrm{C} 3)=213.6^{\circ}$

$\mathrm{C}_{5} \mathrm{O}^{+} \mathrm{A}(\mathrm{C} 1, \mathrm{C} 2, \mathrm{C} 3)=183.9^{\circ}, \mathrm{A}(\mathrm{C} 2, \mathrm{C} 3, \mathrm{C} 4)=160.0^{\circ}, \mathrm{A}(\mathrm{C} 3, \mathrm{C} 4, \mathrm{C} 5)=192.9^{\circ}$

$\mathrm{C}_{7} \mathrm{O}^{+} \mathrm{A}(\mathrm{C} 5, \mathrm{C} 6, \mathrm{C} 7)=165.4^{\circ}$

Table S11. Mulliken spin densities of quartet $\mathrm{C}_{p} \mathrm{O}_{2}^{+}$

\begin{tabular}{|c|c|c|c|c|c|c|c|}
\hline$p^{\mathrm{a}}$ & $\mathrm{O}$ & $\mathrm{C} 1$ & $\mathrm{C} 2$ & $\mathrm{C} 3$ & $\mathrm{C} 4$ & $\mathrm{C} 5$ & $\mathrm{O}^{\prime}$ \\
\hline $2\left(\mathrm{C}_{\mathrm{s}}\right)$ & 0.48 & 0.13 & $\mathbf{1 . 1 7}$ & -- & -- & -- & $\mathbf{1 . 2 3}$ \\
\hline $3\left(\mathrm{C}_{s}\right)$ & 0.52 & 0.10 & $\mathbf{1 . 4 2}$ & 0.38 & -- & -- & 0.59 \\
\hline 4 & $\mathbf{0 . 5 4}$ & 0.12 & $\mathbf{0 . 8 4}$ & $\mathbf{0 . 8 4}$ & 0.12 & -- & -- \\
\hline $5\left(\mathrm{C}_{s}\right)$ & 0.37 & 0.12 & $\mathbf{0 . 7 8}$ & -0.16 & $\mathbf{0 . 9 9}$ & 0.37 & 0.51 \\
\hline 6 & 0.42 & 0.09 & $\mathbf{0 . 7 7}$ & 0.22 & -- & -- & -- \\
\hline 7 & 0.37 & 0.16 & $\mathbf{0 . 5 8}$ & 0.01 & $\mathbf{0 . 7 4}$ & -- & -- \\
\hline
\end{tabular}

${ }^{a}$ Linear $\mathrm{D}_{\infty \mathrm{h}}$ except as noted. Results that are equal by symmetry are shown only once.

Table S12. Optimized bond lengths $(R / p m)$ and dipole moments of quartet $\mathrm{C}_{p} \mathrm{O}_{2}{ }^{+}$

\begin{tabular}{|c|c|c|c|c|c|c|c|}
\hline$p^{\mathrm{a}}$ & $R(\mathrm{C} 1-\mathrm{O})$ & $R(\mathrm{C} 1-\mathrm{C} 2)$ & $R(\mathrm{C} 2-\mathrm{C} 3)$ & $R(\mathrm{C} 3-\mathrm{C} 4)$ & $R(\mathrm{C} 4-\mathrm{C} 5)$ & $R\left(\mathrm{C} p-\mathrm{O}^{\prime}\right)$ & $\mu / \mathrm{D}$ \\
\hline $2(\mathrm{Cs})$ & 114.2 & 133.3 & -- & -- & -- & 133.2 & 0.93 \\
\hline $3(\mathrm{Cs})$ & 114.1 & 132.0 & 144.9 & -- & -- & 118.0 & 1.65 \\
\hline 4 & 115.8 & 128.4 & 134.8 & -- & -- & -- & 0 \\
\hline $5(\mathrm{Cs})$ & 114.3 & 130.7 & 127.8 & 126.3 & 141.1 & 117.2 & 2.02 \\
\hline 6 & 115.3 & 129.0 & 131.0 & 124.6 & -- & -- & 0 \\
\hline 7 & 115.2 & 130.4 & 126.8 & 128.7 & -- & -- & 0 \\
\hline
\end{tabular}

a Linear $\mathrm{D}_{\infty \mathrm{h}}$ except as noted. Results that are equal by symmetry are shown only once. Angles are linear except as noted below:

$\mathrm{C}_{2} \mathrm{O}_{2}{ }^{+} \quad \mathrm{A}(\mathrm{O}, \mathrm{C} 1, \mathrm{C} 2)=189.8^{\circ}, \mathrm{A}\left(\mathrm{C} 1, \mathrm{C} 2, \mathrm{O}^{\prime}\right)=137.2^{\circ}$

$\mathrm{C}_{3} \mathrm{O}_{2}{ }^{+} \quad \mathrm{A}(\mathrm{O}, \mathrm{C} 1, \mathrm{C} 2)=175.3^{\circ}, \mathrm{A}\left(\mathrm{C} 2, \mathrm{C} 3, \mathrm{O}^{\prime}\right)=120.3^{\circ}$

$\mathrm{C}_{5} \mathrm{O}_{2}{ }^{+} \mathrm{A}(\mathrm{C} 3, \mathrm{C} 4, \mathrm{C} 5)=193.0^{\circ} \mathrm{A}\left(\mathrm{C} 4, \mathrm{C} 5, \mathrm{O}^{\prime}\right)=132.1^{\circ}$

Table S13. Monocarbon-insertion energetics ${ }^{\mathrm{a}}$ for doublet $\mathrm{C}_{p} \mathrm{O}_{3}{ }^{+}$.

\begin{tabular}{|r|r|r|}
\hline$p$ & \multicolumn{1}{|c|}{$\Delta_{1} E$} & $\Delta_{1} G_{298}{ }^{\circ}$ \\
\hline 4 & 48.4 & -4.3 \\
\hline 5 & 9.0 & -46.5 \\
\hline 6 & 45.6 & -12.8 \\
\hline
\end{tabular}

${ }^{\mathrm{a}} \mathrm{kJ} / \mathrm{mol}$ 
Table S14. Computed $\Delta E_{\mathrm{TS}}$ between optimized triplets and singlets of neutral oligocarbon monoxides and dioxides (as Fig 3)

\begin{tabular}{|c|c|c|}
\hline $\mathrm{C}_{p} \mathrm{O}_{q}$ & \multicolumn{2}{|c|}{$\left(E_{\mathrm{T}}-E_{\mathrm{S}}\right) / \mathrm{eV}$} \\
\hline$p$ & $q=1$ & $q=2$ \\
\hline 2 & -1.087 & -- \\
\hline 3 & 2.976 & 3.238 \\
\hline 4 & -0.698 & -0.558 \\
\hline 5 & 2.300 & 2.679 \\
\hline 6 & -0.523 & -0.447 \\
\hline 7 & 1.874 & 1.851 \\
\hline
\end{tabular}

Table S15. Computed vertical electron-attachment and CT (Equation 10) energies for $\mathrm{C}_{p} \mathrm{O}^{+}$giving neutral $\mathrm{C}_{p} \mathrm{O}$ in most-stable spin state (Compare Table 14)

\begin{tabular}{|c|c|r|r|}
\hline$p$ & $2 \mathrm{~S}+1$ & $-\Delta_{\mathrm{EA}} E / \mathrm{eV}^{\mathrm{a}}$ & $\Delta_{10} E / \mathrm{eV}^{\mathrm{b}}$ \\
\hline 1 & 1 & 14.156 & -3.323 \\
\hline 2 & 3 & 10.921 & -0.088 \\
\hline 3 & 1 & 10.812 & 0.021 \\
\hline 4 & 3 & 9.769 & 1.064 \\
\hline 5 & 1 & 9.909 & 0.924 \\
\hline 6 & 3 & 8.960 & 1.873 \\
\hline 7 & 1 & 9.311 & 1.522 \\
\hline
\end{tabular}

${ }^{a}$ values as differences between optimized $E$ of cation and of neutral $\mathrm{C}_{p} \mathrm{O}$ at geometry of cation

${ }^{\mathrm{b}} \Delta_{10} E=I\left(\mathrm{C}_{3} \mathrm{O}_{2}\right)+\Delta_{\mathrm{EA}} E\left(\mathrm{C}_{p} \mathrm{O}^{+}\right)$where $I\left(\mathrm{C}_{3} \mathrm{O}_{2}\right)=10.833 \mathrm{eV}$

Table S16. Optimized bond lengths $(R / \mathrm{pm})$ and dipole moments of neutral $\mathrm{C}_{p} \mathrm{O}$ molecules

\begin{tabular}{|c|c|c|c|c|c|c|c|c|c|}
\hline$p^{\mathrm{a}}$ & $R(\mathrm{C} 1-\mathrm{O})$ & $R(\mathrm{C} 1-\mathrm{C} 2)$ & $R(\mathrm{C} 2-\mathrm{C} 3)$ & $R(\mathrm{C} 3-\mathrm{C} 4)$ & $R(\mathrm{C} 4-\mathrm{C} 5)$ & $R(\mathrm{C} 5-\mathrm{C} 6)$ & $R(\mathrm{C} 6-\mathrm{C} 7)$ & $\mu / \mathrm{D}$ & $q / e^{b}$ \\
\hline 2 & 116.2 & 135.5 & -- & -- & -- & -- & -- & 1.34 & 0.112 \\
\hline 3 & 114.9 & 129.5 & 126.7 & -- & -- & -- & -- & 2.27 & 0.129 \\
\hline 4 & 116.3 & 128.3 & 128.2 & 130.4 & -- & -- & -- & 2.63 & 0.110 \\
\hline 5 & 115.6 & 128.3 & 125.9 & 129.0 & 127.4 & -- & -- & 3.57 & 0.120 \\
\hline 6 & 116.3 & 128.1 & 127.4 & 127.3 & 128.7 & 129.3 & -- & 3.77 & 0.105 \\
\hline 7 & 115.8 & 128.1 & 126.4 & 128.0 & 126.2 & 129.1 & 127.6 & 4.76 & 0.113 \\
\hline
\end{tabular}

${ }^{a}$ Each is linear. Values are shown for the most stable spin state: ${ }^{1} \Sigma$ for odd $p,{ }^{3} \Sigma$ for even $p$.

${ }^{\mathrm{b}}$ Magnitude of effective charge on terminal atoms calculated from $\mu$ and total $R$ (See Section 3.5.1)

Table S17. Mulliken spin densities of triplet $\mathrm{C}_{p} \mathrm{O}$ molecules

\begin{tabular}{|c|c|c|c|c|c|c|c|c|}
\hline$p^{\mathrm{a}}$ & $\mathrm{O}$ & $\mathrm{C} 1$ & $\mathrm{C} 2$ & $\mathrm{C} 3$ & $\mathrm{C} 4$ & $\mathrm{C} 5$ & $\mathrm{C} 6$ & $\mathrm{C7}$ \\
\hline 2 & 0.48 & 0.32 & $\mathbf{1 . 2 0}$ & -- & -- & -- & -- & -- \\
\hline $3\left(\mathrm{C}_{s}\right)$ & 0.24 & 0.48 & 0.06 & $\mathbf{1 . 2 2}$ & -- & -- & -- & -- \\
\hline 4 & 0.36 & 0.24 & 0.33 & 0.20 & $\mathbf{0 . 8 7}$ & -- & -- & -- \\
\hline $5\left(\mathrm{C}_{s}\right)$ & 0.27 & 0.21 & 0.30 & 0.28 & 0.26 & $\mathbf{0 . 6 8}$ & -- & -- \\
\hline 6 & 0.28 & 0.19 & 0.30 & 0.15 & 0.25 & 0.15 & $\mathbf{0 . 6 8}$ & -- \\
\hline $7\left(\mathrm{C}_{s}\right)$ & 0.24 & 0.18 & 0.23 & 0.17 & 0.22 & 0.17 & 0.16 & $\mathbf{0 . 6 4}$ \\
\hline
\end{tabular}

${ }^{a}$ Linear $\mathrm{C}_{\infty \mathrm{v}}$ unless otherwise noted 
Table S18. Computed vertical electron-attachment and CT (Equation 10) energies for $\mathrm{C}_{p} \mathrm{O}_{2}{ }^{+}$ giving neutral $\mathrm{C}_{p} \mathrm{O}_{2}$ in most-stable spin state (Compare Table 15)

\begin{tabular}{r|c|r|r|}
\hline$p$ & $2 \mathrm{~S}+1$ & $-\Delta_{\mathrm{EA}} E / \mathrm{eV}^{\mathrm{a}}$ & $\Delta_{10} E / \mathrm{eV}^{\mathrm{b}}$ \\
\hline 1 & 1 & 13.792 & -2.959 \\
\hline 2 & 3 & 8.996 & 1.837 \\
\hline 3 & 1 & 10.587 & 0.246 \\
\hline 4 & 3 & 8.564 & 2.269 \\
\hline 5 & 1 & 9.344 & 1.489 \\
\hline 6 & 3 & 7.993 & 2.840 \\
\hline 7 & 1 & 8.604 & 2.229 \\
\hline
\end{tabular}

a values as differences between optimized $E$ of cation and of neutral $\mathrm{C}_{p} \mathrm{O}_{2}$ at geometry of cation

${ }^{\mathrm{b}} \Delta_{10} E=I\left(\mathrm{C}_{3} \mathrm{O}_{2}\right)+\Delta_{\mathrm{EA}} E\left(\mathrm{C}_{p} \mathrm{O}_{2}{ }^{+}\right)$where $I\left(\mathrm{C}_{3} \mathrm{O}_{2}\right)=10.833 \mathrm{eV}$

Table S19. Mulliken spin densities of triplet $\mathrm{C}_{p} \mathrm{O}_{2}$ molecules

\begin{tabular}{|c|c|c|c|c|c|c|}
\hline$p^{\mathrm{a}}$ & $\mathrm{O}$ & $\mathrm{C} 1$ & $\mathrm{C} 2$ & $\mathrm{C} 3$ & $\mathrm{C} 4$ & $\mathrm{O}^{\prime}$ \\
\hline 2 & 0.56 & 0.44 & -- & -- & -- & -- \\
\hline $3\left(\mathrm{C}_{1}\right)$ & 0.44 & 0.53 & 0.58 & 0.15 & -- & 0.31 \\
\hline 4 & 0.38 & 0.31 & 0.32 & -- & -- & -- \\
\hline $5\left(\mathrm{C}_{2 \mathrm{v}}\right)$ & 0.30 & 0.24 & 0.34 & 0.24 & -- & -- \\
\hline 6 & 0.29 & 0.22 & 0.28 & 0.21 & -- & -- \\
\hline 7 & 0.24 & 0.19 & 0.23 & 0.21 & 0.25 & -- \\
\hline
\end{tabular}

${ }^{a}$ Linear $\mathrm{D}_{\infty \mathrm{h}}$ unless otherwise noted. Results that are equal by symmetry are shown only once.

Table S20. Computed vertical electron-attachment and CT (Equation 10) energies for $\mathrm{C}_{p} \mathrm{O}_{3}{ }^{+}$ giving neutral $\mathrm{C}_{p} \mathrm{O}_{3}$ in the indicated spin state (Compare Table 17)

\begin{tabular}{|c|c|c|c|}
\hline$p$ & $2 \mathrm{~S}+1$ & $-\Delta_{\mathrm{EA}} E / \mathrm{eV}^{\mathrm{a}}$ & $\Delta_{10} E / \mathrm{eV}^{\mathrm{b}}$ \\
\hline \multirow{2}{*}{4} & 1 & 7.432 & 3.401 \\
\cline { 2 - 4 } & 3 & 6.590 & 4.243 \\
\hline \multirow{2}{*}{5} & 1 & 7.484 & 3.349 \\
\cline { 2 - 4 } & 3 & 6.749 & 4.084 \\
\hline \multirow{2}{*}{6} & 1 & 6.967 & 3.866 \\
\cline { 2 - 4 } & 3 & 6.395 & 4.438 \\
\hline 7 & 3 & 6.476 & 4.357 \\
\hline
\end{tabular}

${ }^{a}$ values as differences between optimized $E$ of cation and of neutral $\mathrm{C}_{p} \mathrm{O}_{3}$ at geometry of cation

${ }^{\mathrm{b}} \Delta_{10} E=I\left(\mathrm{C}_{3} \mathrm{O}_{2}\right)+\Delta_{\mathrm{EA}} E\left(\mathrm{C}_{p} \mathrm{O}_{3}{ }^{+}\right)$where $/\left(\mathrm{C}_{3} \mathrm{O}_{2}\right)=10.833 \mathrm{eV}$

Table S21. Mulliken spin densities of triplet $\mathrm{C}_{p} \mathrm{O}_{3}$ molecules

\begin{tabular}{|c|c|c|c|c|c|c|c|c|c|c|}
\hline$p$ & $\mathrm{O}\left(\mathrm{C1}^{\prime}\right)$ & $\mathrm{C1}^{\prime}$ & $\mathrm{O}(\mathrm{C} 1)$ & $\mathrm{C} 1$ & $\mathrm{C} 2$ & $\mathrm{C} 3$ & $\mathrm{C} 4$ & $\mathrm{C} 5$ & $\mathrm{C} 6$ & $\mathrm{O}[\mathrm{C}(p-1)]$ \\
\hline $4^{\mathrm{a}}$ & 0.26 & $\mathbf{0 . 6 2}$ & 0.26 & $\mathbf{0 . 6 2}$ & 0.02 & 0.13 & -- & -- & -- & 0.09 \\
\hline 5 & 0.27 & $\mathbf{0 . 5 7}$ & 0.14 & 0.19 & -0.01 & $\mathbf{0 . 4 9}$ & 0.10 & -- & -- & 0.26 \\
\hline 6 & 0.28 & $\mathbf{0 . 7 0}$ & 0.06 & 0.13 & -0.05 & $\mathbf{0 . 4 7}$ & 0.02 & 0.24 & -- & 0.17 \\
\hline 7 & 0.28 & $\mathbf{0 . 6 2}$ & 0.04 & 0.05 & 0.09 & 0.28 & 0.01 & 0.33 & 0.09 & 0.22 \\
\hline
\end{tabular}

${ }^{\mathrm{a}}$ For $p=4, \mathrm{C} 1^{\prime}-\mathrm{O}$ and $\mathrm{C} 1-\mathrm{O}$ groups are equivalent by $\mathrm{C}_{2 \mathrm{v}}$ symmetry. 